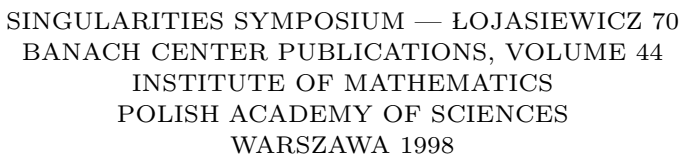

\title{
FELIX KLEIN'S PAPER ON REAL FLEXES VINDICATED
}

\author{
FELICE RONGA \\ Section de Mathématiques, Université de Genève \\ 2-4- rue du Lièvre, CH-1211 Genève 24, Switzerland \\ E-mail: Ronga@math.unige.ch,Web: http:/www.unige.ch/math/
}

Dedicated to Professor Eojasiewicz with gratitude

\begin{abstract}
In a paper written in 1876 [4], Felix Klein gave a formula relating the number of real flexes of a generic real plane projective curve to the number of real bitangents at non-real points and the degree, which shows in particular that the number of real flexes cannot exceed one third of the total number of flexes. We show that Klein's arguments can be made rigorous using a little of the theory of singularities of maps, justifying in particular his resort to explicit examples.
\end{abstract}

0. Introduction. Recently, there has been a renewed interest for enumerative problems over the reals in algebraic geometry [1], which were abandoned after a few attempts by the founders of modern algebraic geometry.

In general, in algebraic geometry, the number of solutions of an enumerative problem over the reals is bounded by the number of solutions over the complex. So, two natural questions arise:

1) Is it possible to arrange that all the solutions are real? If it is the case, the problem is called fully real in [6].

2) Which intermediate number of solutions can be obtained?

Examples.

i) According to Bézout's theorem, two plane curves in general position, of degree $m$ and $n$ respectively intersect in $m \cdot n$ points. By taking curves consisting of lines, some of them real, some pairwise complex conjugate, one can realize any number of intersection points of the form

$$
m \cdot n-2 k, \quad \text { with } 0 \leq 2 k \leq m \cdot n
$$

1991 Mathematics Subject Classification: Primary 14N10; Secondary 58C27.

Received by the editors: September 25, 1996, in the revised form: April 11, 1997.

The paper is in final form and no version of it will be published elsewhere. 
ii) There are 27 lines on a smooth, complex cubic surface. Over the reals, there can be $27,15,7$ or 3 lines [2].

iii) Using Weierstrass form $y^{2}-x(x-1)(x-\alpha)=0$ for the equation of a smooth cubic plane curve, one sees easily that there are always exactly 3 real flexes, whereas there are 9 over the complex.

Inspired by a paper of Zeuthen [10], F. Klein [4] proves that for a real, smooth, generic curve of degree $n$, one has

THEOREM I.

$$
w^{\prime}+2 t^{\prime \prime}=n(n-2)
$$

where $w^{\prime}$ denotes the number of real flexes and $t^{\prime \prime}$ the number of real bitangents at a pair of complex conjugate points (or bitangents of type $t^{\prime \prime}$ for short - we have adopted Klein's notation).

In particular, there are at most $n(n-2)$ real flexes, whereas there are $3 n(n-2)$ over the complex. Klein also gives a formula for generic curves with ordinary cusps and double points, that we will not consider here.

Klein's method consists in deforming a given curve into a smooth, generic one with $n(n-2)$ flexes and no bitangent of type $t^{\prime \prime}$. During the deformation, non-generic curves are encountered, in the neighborhood of which $w^{\prime}$ and $t^{\prime \prime}$ may change. He proves that they do change only when passing through a curve with a degenerate flex, a point at which the tangent intersects the curve with multiplicity 4 . When crossing the stratum of such curves, on one side there are curves having a real line bitangent at two close real points, between which there are two real flexes. Coming close to the stratum, the two tangency points and the two flexes come to coincide, and when moving to the other side, the two flexes disappear, and the two points of tangency become imaginary (an explicit example of such a deformation can be obtained by taking the images by the map $(x, y) \mapsto\left(x^{2}, y\right)$ of circles of diameter 1 centered at the point $(t, 0)$, when $t$ varies from $1 / 4$ to $3 / 4-$ see Figure 1). During such a deformation, $w^{\prime}$ decreases by 2 and $t^{\prime \prime}$ increases by 1 , and therefore $w^{\prime}+2 t^{\prime \prime}$ remains unchanged. This is in fact the key argument.
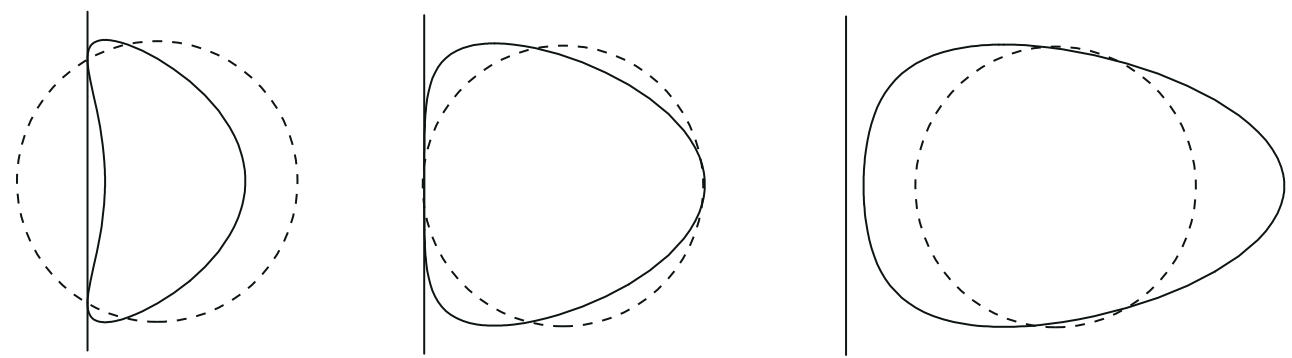

Figure 1. Two flexes disappear and a bitangent of type $t^{\prime \prime}$ appears

The curve with the maximal number of real flexes is obtained as a small deformation of a curve consisting of conics if $n$ is even, conics plus a cubic if $n$ is odd.

In [8], O. Ya. Viro proves a generalized Klein's formula using integration with respect to Euler characteristic, and a similar formula is proved in [9] by C. T. C. Wall, by using in an essential way transcendental topology. 
Klein's approach is still of interest, because it provides examples of curves where the maximal number of $n(n-2)$ real flexes is reached. In addition, his method can be used to show

Theorem II. Curves with any admissible number of real flexes exist:

$$
w^{\prime}=n(n-2)-2 k, \quad w^{\prime} \leq n(n-2), \quad \begin{cases}w^{\prime} \geq 0 & \text { if } n \text { is even } \\ w^{\prime} \geq 3 & \text { if } n \text { is odd. }\end{cases}
$$

Our goal is to interpret Klein's arguments in terms of assertions on the singularities of the universal map naturally associated to the incidence relation of a curve of degree $n$ and a line (see Section 2). In particular, the key argument reduces essentially to observe that on the celebrated swallow-tail singular locus ([7], page 82 and our Figure 2), the curve of double points has two parts, one where two real sheets of the surface intersect, and another, which is a continuation of the previous, but is usually not drawn, where two imaginary sheets of the surface intersect; the two parts are separated by the most singular point of the surface, where the curve of double points and the cuspidal edges meet.

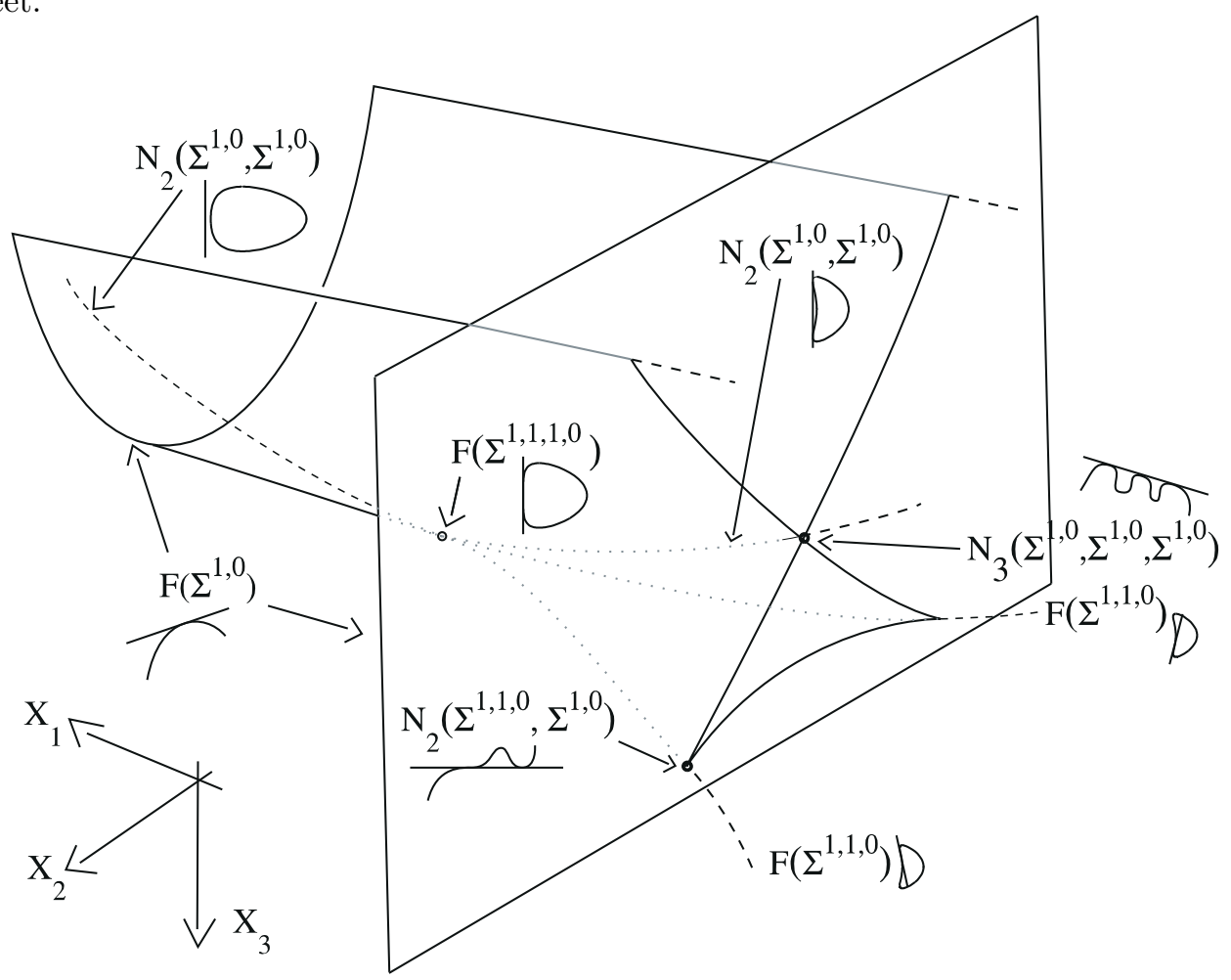

Figure 2. A freehand sketch of the swallow tail and the interpretation of the various strata in terms of the fibers of the map $\theta$

1. First strata. We begin by recalling some general facts about the singularities of maps that we shall use. We refer to [3] for the material needed, but will recall all the basic facts. 
The singular loci $\Sigma^{1_{k}}$. We work simultaneously in the real and complex case. Let $U \subset \mathbb{C}^{n}$ be an open subset and $f: U \rightarrow \mathbb{C}^{n}$ be a holomorphic map, or $U \subset \mathbb{R}^{n}$ and $f: U \rightarrow \mathbb{R}^{n}$ a $\mathcal{C}^{\infty}$ map. We set

$$
\Sigma^{1}(f)=\left\{x \in U \mid \operatorname{dim}\left(\operatorname{Ker}\left(d f_{x}\right)\right)=1\right\} .
$$

If $x^{0} \in \Sigma^{1}(f)$, using the implicit function theorem we may assume that after a change of coordinates in the source and target of $f$, it can be written as

$$
f\left(x_{1}, \ldots, x_{n}\right)=\left(x_{1}, \ldots, x_{n-1}, g\left(x_{1}, \ldots, x_{n}\right)\right)
$$

with $\frac{\partial g}{\partial x_{n}}\left(x^{0}\right)=0$. We shall say that $f$ is $\Sigma^{1}$-transversal if

$$
\exists i_{1} \in\{1, \ldots, n\} \text { such that } \frac{\partial^{2} g}{\partial x_{i_{1}} \partial x_{n}}\left(x^{0}\right) \neq 0 .
$$

Note that the equation of $\Sigma^{1}(f)$ near $x^{0}$ is

$$
\frac{\partial g}{\partial x_{n}}(x)=0
$$

and therefore $\Sigma^{1}$-transversal means that the above equation is of maximal rank, and hence it defines a smooth hypersurface near $x^{0}$.

We define now the notion of $\Sigma^{1_{k}}$-transversal and the flag of subvarieties

$$
\Sigma^{1_{k}}(f) \subset \Sigma^{1_{k-1}}(f) \subset \ldots \subset \Sigma^{1}(f) \subset U
$$

where the symbol $1_{k}$ is an abbreviation for $\underbrace{1, \ldots, 1}_{k}$. We set

$$
\Sigma^{1_{k}}(f)=\left\{x \in U \mid \frac{\partial^{\ell} g}{\partial x_{n}^{\ell}}(x)=0 \quad \forall \ell=1, \ldots, k\right\}
$$

and say that $f$ is $\Sigma^{1_{k}}(f)$-transversal if the above set of equations is of maximal rank.

Note that $\operatorname{Ker}\left(d f_{x^{0}}\right)$ equals the $x_{n^{-a x i s}}$, and therefore $\frac{\partial^{2} f}{\partial x_{n}^{2}}\left(x^{0}\right)=0$ if and only if $\operatorname{dim}\left(\operatorname{Ker}\left(d\left(f \mid \Sigma^{1}(f)\right)\right)\right)=1$, and similarly

$$
\Sigma^{1_{k}}(f)=\left\{x \in \Sigma^{1_{k-1}}(f) \mid \operatorname{dim}\left(\operatorname{Ker}\left(d\left(f \mid \Sigma^{1_{k-1}}(f)\right)\right)\right)=1\right\} .
$$

Finally, we set

$$
\Sigma^{1_{k}, 0}(f)=\Sigma^{1_{k}}(f) \backslash \Sigma^{1_{k+1}}(f) .
$$

If $f: X^{n} \rightarrow Y^{n}$ is now a proper $\mathcal{C}^{\infty}$ or holomorphic map between smooth manifolds, the singular loci $\Sigma^{1_{k}}(f) \subset X$ and the $\Sigma^{1_{k}}$-transversality can be defined with the previous definitions in local coordinates. If $f$ is $\Sigma^{1_{k}}$-transversal for all $k$, then we have a flag of smooth subvarieties:

$$
\Sigma^{1_{n}}(f) \subset \Sigma^{1_{n-1}}(f) \subset \ldots \subset \Sigma^{1}(f) \subset X
$$

with $\Sigma^{1_{k}}(f)$ a smooth submanifold of codimension $k$ of $X, \Sigma^{1_{k}}(f)=\emptyset$ for $k>n$ and

$$
\Sigma^{1_{k}}(f)=\left\{x \in \Sigma^{1_{k-1}}(f) \mid \operatorname{dim}\left(\operatorname{Ker}\left(d\left(f \mid \Sigma^{1_{k}}(f)\right)_{x}\right)\right)=1\right\} .
$$

A more formal and conceptual approach can be found for example in [3], Part II, $\S 3$ (our $\Sigma^{1_{k}, 0}$ is written $S_{1_{k}}$ there). It was proved by B. Morin (see [3], Part II, Theorem 4.1) that 
if $f: X \rightarrow Y$ is $\Sigma^{1_{k}}$-transversal and $x^{0} \in \Sigma^{1_{k}, 0}(f)$, then there exist coordinates systems on $X$ and $Y$, sending $x^{0}$ and $f\left(x^{0}\right)$ to the origin, in which $f$ has the form

$$
f\left(x_{1}, \ldots, x_{n}\right)=\left(x_{1}, \ldots, x_{n-1}, x_{n}^{k+1}+x_{k-1} x_{n}^{k-1}+x_{k-2} x_{n}^{k-2}+\ldots+x_{1} x_{n}\right) .
$$

Self intersections: the singular loci $M_{h}\left(f, \Sigma^{1_{k_{1}}, 0}, \ldots, \Sigma^{1_{k_{h}}, 0}\right)$. Let $f: X^{n} \rightarrow Y^{n}$ be transversal to $\Sigma^{1_{k}}(f)$ for all $k$. We set

$$
\begin{array}{r}
M_{h}\left(f, \Sigma^{1_{k_{1}}, 0}, \ldots, \Sigma^{1_{k_{h}}, 0}\right)=\left\{x \in \Sigma^{1_{k_{1}}, 0}(f) \mid \exists x^{2} \in \Sigma^{1_{k_{2}}, 0}(f), \ldots, x^{h} \in \Sigma^{1_{k_{h}}, 0}(f),\right. \\
\text { with } \left.x, x^{2}, \ldots, x^{h} \text { all different and } f(x)=f\left(x^{2}\right)=\ldots=f\left(x^{h}\right)\right\}
\end{array}
$$

and shall say that $f$ is $M_{h}\left(\Sigma^{1_{k_{1}}, 0}, \ldots, \Sigma^{1_{k_{h}}, 0}\right)$-transversal if the vector spaces

$$
\operatorname{Im}\left(d f_{x}\left(T \Sigma_{x}^{1_{k_{1}}, 0}\right)\right), \ldots, \operatorname{Im}\left(d f_{x^{h}}\left(T \Sigma_{x^{h}}^{1_{k_{h}}, 0}\right)\right)
$$

are in general position in $T W_{f(x)}$; that is, their intersection has codimension $k_{1}+\ldots+k_{h}$.

More notation: we set

$$
N_{h}\left(f, \Sigma^{1_{k_{1}}, 0}, \ldots, \Sigma^{1_{k_{h}}, 0}\right)=f\left(M_{h}\left(f, \Sigma^{1_{k_{1}}, 0}, \ldots, \Sigma^{1_{k_{h}}, 0}\right)\right)
$$

and denote by

$$
M_{h}^{0}\left(f, \Sigma^{1_{k_{1}}, 0}, \ldots, \Sigma^{1_{k_{h}}, 0}\right)
$$

the subset of $M_{h}\left(f, \Sigma^{1_{k_{1}}, 0}, \ldots, \Sigma^{1_{k_{h}}, 0}\right)$ consisting of points that do not belong to higher self-intersections:

$$
\begin{aligned}
& M_{h}^{0}\left(f, \Sigma^{1_{k_{1}}, 0}, \ldots, \Sigma^{1_{k_{h}}, 0}\right)=\left\{x \in \Sigma^{1_{k_{1}}, 0}(f) \mid \exists x^{2} \in \Sigma^{1_{k_{2}}, 0}(f), \ldots, x^{h} \in \Sigma^{1_{k_{h}}, 0}(f),\right. \\
& \text { with } x, x^{2}, \ldots, x^{h} \text { all different, } f(x)=f\left(x^{2}\right)=\ldots=f\left(x^{h}\right) \\
&\text { and } \left.f\left(x^{\prime}\right)=f(x), x^{\prime} \neq x, x^{1}, \ldots, x^{h} \Rightarrow d f_{x^{\prime}} \text { is bijective }\right\} .
\end{aligned}
$$

Remark 1.1. Consider a map of the form

$$
f: U \rightarrow \mathbb{R}^{n+k}, \quad f(x, u)=(g(x, u), u)
$$

where $x \in \mathbb{R}^{n}, u \in \mathbb{R}^{k}, g: U \rightarrow \mathbb{R}^{n}$, and set $g_{u}(x)=g(x, u)$. It is immediate that if, for a fixed $u, g_{u}$ is $\Sigma^{1_{h}}$-transversal in a neighborhood of $x$ for $h \leq k$, then $f$ is $\Sigma^{1_{h}}$-transversal in a neighborhood of $(x, u)$ for $h \leq k$, and that

$$
\Sigma^{1_{h}}\left(g_{u}\right)=\Sigma^{1_{h}}(f) \cap\left(\mathbb{R}^{n} \times\{u\}\right)
$$

in a neighborhood of $x$. For the self-intersections $M_{h}\left(\Sigma^{1_{k_{1}}, 0}, \ldots, \Sigma^{1_{k_{h}}, 0}\right)$ a similar remark holds.

EXAMPLE 1.2. All the types of singularities and their mutual positions that we shall need are those of codimension at most 3; they can all be contemplated on the following example. Let

$$
F\left(x_{1}, x_{2}, x_{3}\right)=\left(x_{1}, x_{2}, x_{3}^{4}+x_{3}^{2} x_{1}+x_{3} x_{2}\right) .
$$

We have

$$
d F=\left(\begin{array}{ccc}
1 & 0 & 0 \\
0 & 1 & 0 \\
x_{3}^{2} & x_{3} & 4 x_{3}^{3}+2 x_{3} x_{1}+x_{2}
\end{array}\right), \quad \Sigma^{1}(F)=\left\{\left(x_{1}, x_{2}, x_{3}\right) \mid x_{2}=-4 x_{3}^{3}-2 x_{3} x_{1}\right\}
$$




$$
\begin{array}{r}
\Sigma^{1,1}(F)=\left\{\left(x_{1}, x_{2}, x_{3}\right) \in \Sigma^{1} \mid \frac{\partial^{2}\left(x_{3}^{4}+x_{3}^{2} x_{1}+x_{3} x_{2}\right)}{\partial x_{3}^{2}}=0\right\}=\left\{\left(-6 x_{3}^{2}, 8 x_{3}^{3}, x_{3}\right)\right\} \\
\Sigma^{1,1,1,0}(F)=\{0\} .
\end{array}
$$

$\Sigma^{1}(F)$ can be parametrized by $\alpha:\left(x_{1}, x_{3}\right) \mapsto\left(x_{1},-4 x_{3}^{3}-2 x_{3} x_{1}, x_{3}\right)$, and $F\left(\Sigma^{1}(F)\right)$ can be parametrized by

$$
\left(x_{1}, x_{3}\right) \mapsto\left(x_{1},-4 x_{3}^{3}-2 x_{1} x_{3},-3 x_{3}^{4}-x_{1} x_{3}^{2}\right)
$$

and from there one can calculate that

$$
M_{2}\left(F, \Sigma^{1,0}, \Sigma^{1,0}\right)=\alpha\left(\left\{\left(x_{1}, 0, x_{3}\right) \mid x_{1} \neq 0, x_{1}=-2 x_{3}^{2}\right\}\right),
$$

and so its closure $\bar{M}_{2}\left(F, \Sigma^{1,0}, \Sigma^{1,0}\right)$ can be parametrized by $x_{3} \mapsto\left(-2 x_{3}^{2}, 0, x_{3}\right)$. The restriction of $F$ to $\bar{M}_{2}\left(F, \Sigma^{1,0}, \Sigma^{1,0}\right)$ composed with the latter parametrization reads:

$$
x_{3} \mapsto\left(-2 x_{3}^{2}, 0,-x_{3}^{4}\right) .
$$

Also, $F\left(\Sigma^{1,1}(F)\right)$ is parametrized by

$$
x_{3} \mapsto\left(-6 x_{3}^{2}, 8 x_{3}^{3}, 3 x_{3}^{4}\right) .
$$

For a complete picture, we still need to produce the singular strata $M_{2}\left(\Sigma^{1,1,0}, \Sigma^{1,0}\right)$ and $M_{3}\left(\Sigma^{1,0}, \Sigma^{1,0}, \Sigma^{1,0}\right)$. This can be done by taking the disjoint union of $F$ and the map $G\left(u_{1}, u_{2}, u_{3}\right)=\left(u_{1}^{2}-1, u_{2}, u_{3}\right)$. The only singular locus of $G$ is $\Sigma^{1,0}(G)=\left\{\left(0, u_{2}, u_{3}\right)\right\}$, whose image $G\left(\Sigma^{1,0}(G)\right)=\left\{\left(-1, u_{2}, u_{3}\right)\right\}$ meets transversally $M_{2}\left(\Sigma^{1,0}(F), \Sigma^{1,0}(F)\right)$ and $F\left(\Sigma^{1,1,0}(F)\right)$ at $(-1,0,1 / 4)$ and $(-1, \pm 8(1 / \sqrt{6}), \pm 1 / \sqrt{6})$ respectively (see Figure 2 ).

2. The universal incidence relation map for curves of degree $n$ and lines. Let $\mathbb{C}\left[x_{0}, x_{1}, x_{2}\right]_{n}$ and $\mathbb{R}\left[x_{0}, x_{1}, x_{2}\right]_{n}$ denote the vector spaces of homogeneous polynomials of degree $n$ with complex and real coefficients respectively. We denote by

$$
\mathbb{P}_{n, \mathbb{C}}=\mathbb{P}\left(\mathbb{C}\left[x_{0}, x_{1}, x_{2}\right]_{n}\right) \quad \text { and } \quad \mathbb{P}_{n, \mathbb{R}}=\mathbb{P}\left(\mathbb{R}\left[x_{0}, x_{1}, x_{2}\right]_{n}\right)
$$

the associated projective spaces. Let $\mathbb{P}_{\mathbb{C}}^{2}$ and $\mathbb{P}_{\mathbb{R}}^{2}$ denote respectively the complex and real projective plane. For $[f] \in \mathbb{P}_{n, \mathbb{C}}, V(f)=\left\{[x] \in \mathbb{P}_{\mathbb{C}}^{2} \mid f(x)=0\right\}$ denotes the variety defined by $f$.

When we mean either complex or real spaces, we drop the subscripts $\mathbb{C}$ and $\mathbb{R}$.

Denote by $\check{\mathbb{P}}_{\mathbb{C}}^{2}$, respectively $\check{\mathbb{P}}_{\mathbb{R}}^{2}$, the dual projective planes, namely the space of lines of $\mathbb{P}^{2}$, or equivalently $\check{\mathbb{P}}_{\mathbb{C}}^{2}=\mathbb{P}_{1, \mathbb{C}}, \check{\mathbb{P}}_{\mathbb{R}}^{2}=\mathbb{P}_{1, \mathbb{R}}$.

We define the Zariski open subsets of $\mathbb{P}_{n, \mathbb{C}}$ and $\mathbb{P}_{n, \mathbb{R}}$, respectively:

$$
\mathbb{P}_{n, \mathbb{C}}^{0}=\left\{[f] \in \mathbb{P}_{n, \mathbb{C}} \mid V(f) \text { does not contain a line }\right\}, \quad \mathbb{P}_{n, \mathbb{R}}^{0}=\mathbb{P}_{n, \mathbb{C}}^{0} \cap \mathbb{P}_{n, \mathbb{R}}
$$

For $n \geq 3, \mathbb{P}_{n, \mathbb{R}}^{0}$ is connected, because the subspace of $\mathbb{P}_{n, \mathbb{R}}$ of curves containing a line has codimension

$$
\operatorname{dim}\left(\mathbb{P}_{n, \mathbb{R}}\right)-\operatorname{dim}\left(\check{\mathbb{P}}^{2} \times \mathbb{P}_{(n-1), \mathbb{R}}\right)=\frac{n(n+3)}{2}-\left(2+\frac{(n-1)(n+2)}{2}\right)=n-1 .
$$

We assume henceforth that $n \geq 3$, without any loss of flexes.

The universal incidence relation is defined to be the variety

$$
W=\left\{([x],[\phi],[f]) \in \mathbb{P}^{2} \times \check{\mathbb{P}}^{2} \times \mathbb{P}_{n}^{0} \mid \phi(x)=0, f(x)=0\right\}
$$


and the universal incidence relation map

$$
\theta: W \rightarrow \check{\mathbb{P}}^{2} \times \mathbb{P}_{n}^{0}
$$

is the restriction to $W$ of the natural projection $\mathbb{P}^{2} \times \check{\mathbb{P}}^{2} \times \mathbb{P}_{n}^{0} \rightarrow \check{\mathbb{P}}^{2} \times \mathbb{P}_{n}^{0}$. For $[x] \in \mathbb{P}^{2}$ fixed, the set of equations $\phi(x)=0, f(x)=0$ in $\check{\mathbb{P}}^{2} \times \mathbb{P}_{n}^{0}$ is of maximal rank, from which it follows that $W$ is a smooth variety.

Denote by $T: \check{\mathbb{P}}^{2} \times \mathbb{P}_{n}^{0} \rightarrow \mathbb{P}_{n}^{0}$ the natural projection and set $S=T \circ \theta: W \rightarrow \mathbb{P}_{n}^{0}$. We have a commutative diagram of maps:

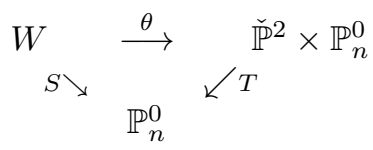

For $[f] \in \mathbb{P}_{n}^{0}$, set

$$
W_{f}=S^{-1}([f]), \quad \theta_{f}=\theta \mid W_{f}: W_{f} \rightarrow \check{\mathbb{P}}^{2} .
$$

We can view the map $\theta$ as the family of maps $\theta_{f},[f] \in \mathbb{P}_{n}^{0}$. The fiber of $\theta_{f}$ over the line $[\phi]$ is constituted by the intersection $V(f) \cap V(\phi)$. Studying the singularities of the map $\theta_{f}$ amounts to study the possible special intersections of a line and the curve $V(f)$ : the theorem below shows that if $V(f)$ is smooth, $\theta_{f}\left(\Sigma^{1}\left(\theta_{f}\right)\right.$ is the dual curve of $V(f)$, $N_{2}\left(\theta_{f}, \Sigma^{1}, \Sigma^{1}\right)$ is the set of bitangents, and $\Sigma^{1,1}\left(\theta_{f}\right)$ identifies to the set of flexes of $V(f)$.

\section{TheOREM 2.1.}

$$
([x],[\phi],[f]) \in \Sigma^{1_{k}, 0}(\theta) \Longleftrightarrow\left\{\begin{array}{l}
\text { the restriction of } f \text { to the line } \ell \text { has a zero } \\
\text { of multiplicity exactly } k+1 \text { at } x .
\end{array}\right.
$$

The map $\theta$ is transversal to the $\Sigma^{1_{k}}$ 's and to the $M_{h}\left(\Sigma^{1_{k_{1}}}, \ldots, \Sigma^{1_{k_{h}}}\right)$ 's.

Proof. We may alternatively consider $\theta$ as a family of maps $\theta_{\ell}: W_{\ell} \rightarrow \mathbb{P}_{n}^{0}$, where $W_{\ell}=\theta^{-1}\left(\{\ell\} \times \mathbb{P}_{n}^{0}\right)$ parametrized by $\ell \in \check{\mathbb{P}}^{2}$ :

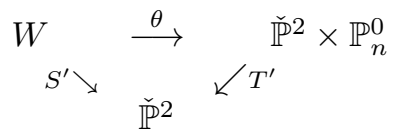

$W_{\ell}$ is smooth, since the natural map $W_{\ell} \rightarrow \check{\mathbb{P}}^{2}$ is a fiber bundle. We will show that for each $\ell, \theta_{\ell}$ has the required transversality properties and interpretation of the singular loci, and the theorem will follow from Remark 1.1. Note that for fixed $\ell, \theta_{\ell}$ is an extension of the universal family of polynomials of degree $n$ on the line $\ell$, as shown by its local expression $\varnothing$ below, and it is therefore no surprise that it satisfies the required transversality properties. Anyway, we give a formal proof now.

Let $z=\left(\left[x^{0}\right], \ell,\left[f^{0}\right]\right) \in W$. First we introduce local coordinates on the various projective spaces and on $W$. We may assume that $\left[x^{0}\right]=[0: 0: 1],\left[\phi^{0}\right]=[0: 1: 0]$. Take affine coordinates $(u, v) \mapsto[u: v: 1]$ near $x^{0}$, then

$$
f^{0}(u, v)=\sum_{0 \leq i, j \leq n} a_{i, j}^{0} u^{i} v^{j}
$$

Since $f^{0}\left(x^{0}\right)=0, a_{0,0}^{0}=0$; since $f^{0}$ does not contain the line $\{[u: 0: 1]\}$ defined by $\phi^{0}$, 
we have

$$
f^{0}(u, 0)=a_{k+1,0}^{0} u^{k+1}+\sum_{k+1<h \leq n} a_{h, 0}^{0} u^{h} \quad \text { with } \quad a_{k+1,0}^{0} \neq 0 \text { for some } k \leq n-1,
$$

and so we may assume that $a_{k+1,0}^{0}=1$. The $\left(a_{i, j}\right)_{0 \leq i, j \leq n,(i, j) \neq(k+1,0)}$ are affine coordinates on $\mathbb{P}_{n}$ near $\left[f^{0}\right]$, that we will write as follows: $\left[a_{0,0}, 1,\left(a_{i, j}\right)_{(i, j) \in I}\right]$, where $I=\{(i, j) \mid 0 \leq i, j \leq n,(i, j) \neq(k+1,0),(0,0)\}$. Define

$$
F\left(u, v,\left(a_{i, j}\right)_{(i, j) \in I}\right)=u^{k+1}+\sum_{(i, j) \in I} a_{i, j} u^{i} v^{j}
$$

so that, if for $u^{0}, v^{0}$ fixed we set $a_{0,0}=-F\left(u^{0}, v^{0}, a_{i, j}\right)$, the polynomial

$$
f(u, v)=a_{0,0}+u^{k+1}+\sum_{(i, j) \in I} a_{i, j} u^{i} v^{j}
$$

vanishes at $\left(u^{0}, v^{0}\right)$. The map

$$
\left(u,\left(a_{i, j}\right)_{(i, j) \in I}\right) \mapsto\left([u: 0: 1],\left[-F\left(u, 0, a_{i, j}\right), 1,\left(a_{i, j}\right)_{(i, j) \in I}\right]\right)
$$

defines local coordinates on $W_{\ell}$ near $z$, using which $\theta_{\ell}$ reads:

$$
\theta_{\ell}^{\text {loc }}:\left(u,\left(a_{i, j}\right)_{(i, j) \in I}\right) \mapsto\left(-u^{k+1}-\sum_{\substack{0 \leq i \leq n, i \neq k+1}} a_{i, 0} u^{i},\left(a_{i, j}\right)_{(i, j) \in I}\right) .
$$

Set

$$
g\left(u,\left(a_{i, j}\right)_{(i, j) \in I}\right)=-u^{k+1}-\sum_{0 \leq i \leq n, i \neq k+1} a_{i, 0} u^{i}
$$

Since

$$
\operatorname{Ker}\left(d \theta_{\ell}^{\text {loc }}\left(0, a_{i, j}^{0}\right)\right)=\{(*, 0,0,0)\}
$$

and

$$
\frac{\partial^{\ell+1} g}{\partial a_{\ell, 0} \partial u^{\ell}}\left(0, a_{i, j}^{0}\right)=-\ell !, \ell \leq k, \quad \frac{\partial^{k+1} g}{\partial u^{k+1}}\left(0, a_{i, j}^{0}\right)=-(k+1) !,
$$

we see that $\left(\left[x^{0}\right], \ell,\left[f^{0}\right]\right) \in \Sigma^{1_{k}, 0}\left(\theta_{\ell}\right)$ and that $\theta_{\ell}$ is $\Sigma^{1_{k}, 0}$-transversal.

It follows also that

$$
T\left(\Sigma^{1_{k}}(f)\right)_{z}=\left\{\bar{f}=u^{k+1}+\sum_{k<h \leq n} \bar{a}_{h, 0} u^{h}+\sum_{0 \leq i \leq n, 0<j \leq n} \bar{a}_{i, j} u^{i} v^{j}\right\}
$$

which is the space of polynomials in two variables $u, v$ of degree $n$, with $a_{k+1,0}=1$, whose restriction to $\ell$ vanishes of order at least $k$ at $x$, and transversality to $M_{h}\left(\Sigma^{1_{k_{1}}}, \ldots, \Sigma^{1_{k_{h}}}\right)$ follows from the fact that given distinct $x_{1}, \ldots, x_{h} \in \mathbb{C}$ and positive integers $k_{1}, \ldots, k_{h}$ such that $k_{1}+1+\ldots+k_{h}+1 \leq n$, the linear conditions imposed on polynomials of degree $n$ in one variable to have roots of multiplicity at least $k_{i}+1$ at $x_{i}, i=1, \ldots, h$ are linearly independent.

COROLlaRY 2.2. There exists a non-empty Zariski open subset $\Omega_{n, \mathbb{C}} \subset \mathbb{P}_{n, \mathbb{C}}^{0}$ such that for $f \in \Omega_{n, \mathbb{C}}, \theta_{f}: W_{f} \rightarrow \check{\mathbb{P}}^{2}$ presents only the singularities $\Sigma^{1,0}, \Sigma^{1,1,0}, M_{2}\left(\Sigma^{1,0}, \Sigma^{1,0}\right)$, to which it is transversal. 
Proof. For a smooth subvariety $X \subset W$, denote by $S(X)_{\text {crit }}$ the set of non-regular values of the map $S \mid X: X \rightarrow \mathbb{P}_{n, \mathbb{C}}^{0}$. Set

$$
\begin{aligned}
& K=S\left(\Sigma^{1,0}(\theta)\right)_{\text {crit }} \cup S\left(\Sigma^{1,1,0}(\theta)\right)_{\text {crit }} \cup S\left(\Sigma^{1,1,1,0}(\theta)\right)_{\text {crit }} \cup \\
& \bigcup_{\substack{0<h<n, 0<k_{1}, \ldots, k_{h}<n}} S\left(M_{h}\left(\theta, \Sigma^{1_{k_{1}}, 0}, \ldots, \Sigma^{1_{k_{h}}, 0}\right)\right)_{\text {crit }} .
\end{aligned}
$$

Then $K \subset \mathbb{P}_{n, \mathbb{C}}^{0}$ is algebraic of codimension at least 1 , and we may take

$$
\Omega_{n, \mathbb{C}}=\mathbb{P}_{n, \mathbb{C}}^{0} \backslash K \text {. }
$$

For the real case, we set

$$
\Omega_{n, \mathbb{R}}=\Omega_{n, \mathbb{C}} \cap \mathbb{P}_{n, \mathbb{R}}^{0} .
$$

The set $K$ in the proof of the corollary above is the "catastrophy set" of the family of maps $\theta_{f}: W_{f} \rightarrow \check{\mathbb{P}}_{2} \times \mathbb{P}_{n}^{0}$, and $\Omega_{n}$ is the set of "generic curves". For our purposes, we need to analyze more in detail the set $K$. We do this in the next section.

3. Controlling flexes and bitangents. Let $W_{\mathbb{R}}=W \cap\left(\mathbb{P}_{\mathbb{R}}^{2} \times \check{\mathbb{P}}_{\mathbb{R}}^{2} \times \mathbb{P}_{n, \mathbb{R}}\right), \theta_{\mathbb{R}}=$ $\theta \mid W_{\mathbb{R}}: W_{\mathbb{R}} \rightarrow \check{\mathbb{P}}_{\mathbb{R}}^{2} \times \mathbb{P}_{n, \mathbb{R}}^{0}$.

Consider the map

$$
\phi_{\mathbb{R}}: \Sigma^{1,1}\left(\theta_{\mathbb{R}}\right) \rightarrow \mathbb{P}_{n, \mathbb{R}}^{0}
$$

which is the restriction to $\Sigma^{1,1}\left(\theta_{\mathbb{R}}\right)$ of the projection of $S_{\mathbb{R}}: W_{\mathbb{R}} \rightarrow \mathbb{P}_{n, \mathbb{R}}^{0}$. The fibers $\phi_{\mathbb{R}}^{-1}([f])$ identify to the set of real flexes on $V(f)$, which might be degenerated. The restriction of $\phi_{\mathbb{R}}$ to $\phi_{\mathbb{R}}^{-1}\left(\Omega_{n, \mathbb{R}}\right)$ is a covering map, but $\Omega_{n, \mathbb{R}}$ is not connected. We can view $\phi$ as the "control map" for real flexes; we must analyze how the fibers of $\phi$ change as we move from one connected component of $\Omega_{n, \mathbb{R}}$ to another. Note that it follows from Theorem 2.1 that $\Sigma^{1,1}(\theta)$ is smooth.

For the next propositions we work simultaneously in the real and complex case again. Let $[x] \in \mathbb{P}^{2}$ be an ordinary double point on the curve defined by $f,[\ell]$ a line through $[x]$ simply tangent to one of the two branches of the curve through $x$. Then the intersection of $\ell$ and $V(f)$ at $[x]$ has multiplicity exactly 3 , and so $([x], \ell,[f]) \in \Sigma^{1,1,0}(\theta)$. We denote by $\Delta^{0}$ the set of such $([x], \ell,[f])$ and by $\Delta$ its closure in $W$.

Proposition 3.1. Let $z=([x], \ell,[f]) \in \Sigma^{1,1}(\theta)$.

1) $d \phi_{z}$ is not bijective $\Longleftrightarrow z \in \Delta \cup \Sigma^{1,1,1}(\theta)$.

2) If $z \in \Delta^{0}$, $\phi$ can be written in suitable local coordinates near $z$ as $\left(z_{1}, \ldots, z_{N}\right) \mapsto$ $\left(z_{1}^{3}, z_{2}, \ldots, z_{N}\right)$, and therefore in the real case it is a local homeomorphism at those points.

We first need a lemma, that we work out over the reals, leaving to the reader to check the validity of the complex analogue, in which case $\mathcal{C}^{\infty}$ must be replaced by holomorphic.

Let $U \subset \mathbb{R}^{N}$ be open and $\rho: U \rightarrow \mathbb{R}^{N}$ a $\mathcal{C}^{\infty}$ map. For $x \in U$, the $k$-th derivative of $\rho$ at $x$ is a homogeneous map of degree $k$ :

$$
d^{k} \rho_{x}: \mathbb{R}^{N} \rightarrow \mathbb{R}^{N} .
$$

Consider the composition

$$
d^{k} \widetilde{\rho}_{x}: \operatorname{Ker}\left(d \rho_{x}\right) \subset \mathbb{R}^{N} \stackrel{d^{k} \rho_{x}}{\longrightarrow} \mathbb{R}^{N} \rightarrow \mathbb{R}^{N} / \operatorname{Im}\left(d \rho_{x}\right)=\operatorname{Coker}\left(d \rho_{x}\right) .
$$


It is readily checked that if $d^{h} \widetilde{\rho}_{x}=0,1<h \leq k$, then $d^{k+1} \widetilde{\rho}_{x}$ is intrinsic, which means that it is affected only by the linear part of the local diffeomorphisms near $x$ and $\rho(x)$; the conditions $d^{h} \widetilde{\rho}_{x}=0,1<h \leq k$ also are preserved by coordinate changes. This ensures that the hypothesis in the next lemma are preserved by coordinate changes.

In fact, $d^{k} \widetilde{\rho}_{x}$ is a restriction of the $k$-th intrinsic derivate introduced by Porteous [5].

Lemma 3.2. Let $U \subset \mathbb{R}^{N}$ be open and $\rho: U \rightarrow \mathbb{R}^{N}$ be a $\mathcal{C}^{\infty}$ map. Assume that

1) $\Sigma(\rho)=\left\{x \in U \mid \operatorname{dim}\left(\operatorname{Ker}\left(d \rho_{x}\right)\right)=1\right\}$ is smooth in a neighborhood of $x^{0} \in \Sigma(\rho)$.

2) $d^{h} \widetilde{\rho}_{x}=0, h \leq k, x \in \Sigma(\rho)$, and $d^{k+1} \widetilde{\rho}_{x^{0}} \neq 0$.

Then there exist coordinate systems near $x^{0}$ and $\phi\left(x^{0}\right)$ in which $\rho$ reads:

$$
\left(x_{1}, \ldots, x_{N}\right) \mapsto\left(x_{1}^{k+1}, x_{2}, \ldots, x_{N}\right) .
$$

Proof. We may assume that $x^{0}=0$ and that

$$
\rho\left(x_{1}, \ldots, x_{N}\right)=\left(g\left(x_{1}, \ldots, x_{N}\right), x_{2}, \ldots, x_{N}\right) .
$$

If $x \in \Sigma(\rho), \operatorname{Ker}\left(d \rho_{x}\right)=\{(*, 0, \ldots, 0)\}, \operatorname{Coker}\left(d \rho_{x}\right) \simeq\{(*, 0, \ldots, 0)\}$ and $d^{h} \widetilde{\rho}_{x}$ identifies with $\frac{\partial^{h} g}{\partial x_{1}^{h}}(x)$.

Let $u(x)$ be a local equation of $\Sigma(\rho)$ near 0 . Since $\frac{\partial^{k} g}{\partial x_{1}^{k}}(x)=0$ for $x \in \Sigma(\rho)$, there exists a function $v(x)$ in a neighborhood of 0 such that $\frac{\partial^{k} g}{\partial x_{1}^{k}}(x)=u(x) v(x)$, and so $\frac{\partial^{k+1} g}{\partial x_{1}^{k+1}}(x)=$ $\frac{\partial u}{\partial x_{1}}(x) v(x)+u(x) \frac{\partial v}{\partial x_{1}}(x)$. Evaluating at $x=0$ shows that $\frac{\partial^{k+1} g}{\partial x_{1}^{k+1}}(0)=\frac{\partial u}{\partial x_{1}}(0) v(0)$, which implies that $\frac{\partial u}{\partial x_{1}}(0) \neq 0$. We can therefore choose $x_{1}^{\prime}=u(x)$ as a new first coordinate. In the new coordinates, again denoted by $x_{1}, \ldots, x_{N}$, the local equation of $\Sigma(\rho)$ near 0 is $x_{1}=0$ and so

$$
\frac{\partial^{h} g}{\partial x_{1}^{h}}\left(0, x_{2}, \ldots, x_{N}\right)=0,1 \leq h \leq k, \quad \frac{\partial^{k+1} \rho}{\partial x_{1}^{k+1}}(0) \neq 0
$$

Therefore $g\left(x_{1}, \ldots, x_{N}\right)=x_{1}^{k+1} \cdot g_{1}\left(x_{1}, \ldots, x_{N}\right)$, with $g_{1}(0) \neq 0$. After changing perhaps the sign of the first coordinate in the target, we may assume that $g_{1}(0)>0$. If we take the new coordinate

$$
x_{1}^{\prime}=x_{1}\left(g_{1}\left(x_{1}, \ldots, x_{N}\right)\right)^{1 / k}
$$

then $\rho$ has the desired form.

Proof of Proposition 3.1. Let $E=\mathbb{R}^{2}$ or $\mathbb{C}^{2}, \ell_{0} \subset E$ be a line and let $\ell^{\prime}$ be distinct from $\ell, \ell_{0} \cap \ell^{\prime}=\left\{x_{0}\right\}$. Then the lines in a neighborhood of $\ell_{0}$ can be viewed as graphs of affine maps $\ell: \ell_{0} \rightarrow \ell^{\prime}$. Any vector $x \in E$ can be uniquely decomposed as $x=x^{0}+x^{\prime}$, where $x^{0} \in \ell_{0}, x^{\prime} \in \ell^{\prime}$. Denote by $i_{\ell_{0}}: \ell_{0} \rightarrow E$ the natural inclusion and by $L\left(\ell_{0}, \ell^{\prime}\right)$ the space of affine maps from $\ell_{0}$ to $\ell^{\prime}$; then the line corresponding to $\ell \in L\left(\ell_{0}, \ell^{\prime}\right)$ is the image of the map $i_{\ell_{0}}+\ell: \ell_{0} \rightarrow E$, and $x \in E$ is on that line if and only if $x^{\prime}=\ell\left(x^{0}\right)$.

Let $z=\left(\left[x_{0}\right], \ell_{0},[f]\right) \in W$. Using the coordinates $E \times L\left(\ell_{0}, \ell^{\prime}\right) \times U$, where $U$ is some affine coordinate system containing $[f]$, we have the following equations for our various 
spaces:

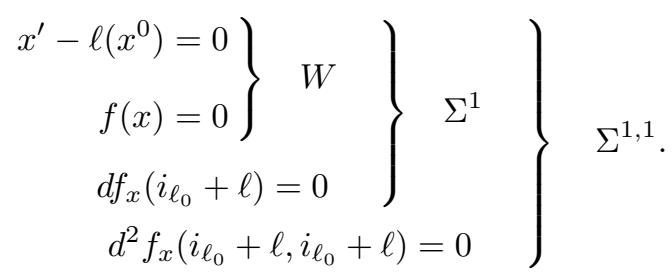

Denoting tangent vectors with overlined symbols, we have the following equations for the corresponding tangent spaces at $z$ :

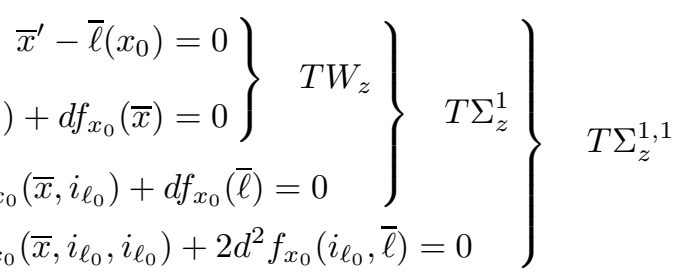

and $\operatorname{Ker}\left(d \phi_{z}\right)$ is the set of tangent vectors to $\Sigma^{1,1}$ of the form $(\bar{x}, \bar{\ell}, 0)$, that is

$$
\begin{aligned}
\operatorname{Ker}\left(d \phi_{z}\right)=\left\{(\bar{x}, \bar{\ell}, 0) \mid \bar{x}^{\prime}-\bar{\ell}\left(x_{0}^{0}\right)=0, d f_{x_{0}}(\bar{x})\right. & =0, d^{2} f_{x_{0}}\left(\bar{x}, i_{\ell_{0}}\right)+d f_{x_{0}}(\bar{\ell})=0, \\
& \left.d^{3} f_{x_{0}}\left(\bar{x}, i_{\ell_{0}}, i_{\ell_{0}}\right)+2 d^{2} f_{x_{0}}\left(i_{\ell_{0}}, \bar{\ell}\right)=0\right\} .
\end{aligned}
$$

If $d f_{x_{0}} \neq 0$, then $d f_{x_{0}}(\bar{x})=0 \Rightarrow \bar{x} \in \ell_{0} \Rightarrow \bar{x}^{\prime}=0 \Rightarrow \bar{\ell}\left(x_{0}^{0}\right)=0$ and $d f_{x_{0}}(\bar{\ell})=0 \Rightarrow \bar{\ell}=0$. But then $d^{3} f\left(\bar{x}, i_{\ell_{0}}, i_{\ell_{0}}\right)=0$ with $\bar{x} \in \ell_{0}$ not zero is possible only if $z \in \Sigma^{1,1,1}(\theta)$, that is $d^{3} f_{x_{0}}\left(i_{\ell_{0}}, i_{\ell_{0}}, i_{\ell_{0}}\right)=0$.

If $x$ is an ordinary double point on $f$, then $d f_{x}=0$, and if $\ell_{0}$ is simply tangent to one of the branches through $x_{0}$, then $d^{2} f_{x}\left(\bar{x}, i_{\ell_{0}}\right)=0 \Rightarrow \bar{x} \in \ell_{0} \Rightarrow \bar{\ell}\left(x_{0}\right)=0$ and $d^{3} f\left(i_{\ell_{0}}, i_{\ell_{0}}, i_{\ell_{0}}\right) \neq 0$. Thus

$$
\operatorname{Ker}\left(d \phi_{z}\right)=\left\{(\bar{x}, \bar{\ell}, 0) \mid \bar{x} \in \ell_{0}, \bar{\ell}\left(x_{0}\right)=0, d^{3} f_{x_{0}}\left(\bar{x}, i_{\ell_{0}}, i_{\ell_{0}}\right)+2 d^{2} f_{x_{0}}\left(i_{\ell_{0}}, \bar{\ell}\right)=0\right\}
$$

and the projection $(\bar{x}, \bar{\ell}) \mapsto \bar{x}$ induces an isomorphism from $\operatorname{Ker}\left(d \phi_{z}\right)$ to $\ell_{0}$. If $\bar{f} \in$ $\operatorname{Im}\left(d \phi_{z}\right)$, then it follows from equation $(\star)$ above that $\bar{f}\left(x_{0}\right)=0 . \operatorname{But} \operatorname{dim}\left(\operatorname{Ker}\left(d \phi_{z}\right)\right)=1$, hence we have exactly

$$
\operatorname{Im}\left(d \phi_{z}\right)=\left\{\bar{f} \mid \bar{f}\left(x_{0}\right)=0\right\}
$$

and so the intrinsic derivatives of $\phi$ at $z$ identify with the ordinary derivatives of $f$ at $x_{0}$ restricted to $\ell_{0}$ :

$$
(\bar{x}, \bar{\ell}) \in \operatorname{Ker}\left(d \phi_{z}\right), \quad d^{h} \widetilde{\phi}_{z}(\bar{x}, \bar{\ell})=d^{3} f_{x_{0}}(\bar{x}) .
$$

Therefore $d^{2} \widetilde{\phi}_{z}=0, d^{3} \widetilde{\phi}_{z} \neq 0$ and we can apply Lemma 3.2.

Let $\bar{M}_{2}(\theta)=\bar{M}_{2}\left(\theta, \Sigma^{1,0}, \Sigma^{1,0}\right)$ be the closure of $M_{2}\left(\theta, \Sigma^{1,0}, \Sigma^{1,0}\right)$ in $W$. As "control map" for bitangents we take the restriction

$$
\psi: \bar{M}_{2} \rightarrow \mathbb{P}_{n}^{0}
$$

of the natural projection $S: W \rightarrow \mathbb{P}_{n}^{0} . \bar{M}_{2}$ is not smooth, so in this case we must understand where it is not smooth and near which smooth points it might not be a local homeomorphism. 
Proposition 3.3. There is a decomposition

$$
\begin{aligned}
\bar{M}_{2}(\theta)=M_{2}^{0}\left(\theta, \Sigma^{1,0}, \Sigma^{1,0}\right) \cup M_{2}^{0}\left(\theta, \Sigma^{1,1,0}, \Sigma^{1,0}\right) \cup M_{2}^{0}\left(\theta, \Sigma^{1,0}, \Sigma^{1,1,0}\right) \\
\\
\cup M_{3}^{0}\left(\theta, \Sigma^{1,0}, \Sigma^{1,0}, \Sigma^{1,0}\right) \cup \Sigma^{1,1,1,0}(\theta) \cup K_{2}
\end{aligned}
$$

where $K_{2}$ is an algebraic subset of codimension 2 in $\bar{M}_{2}$, and all the other strata, except $M_{2}^{0}\left(\theta, \Sigma^{1,0}, \Sigma^{1,0}\right)$, have codimension 1 .

$\bar{M}_{2}$ is smooth at points of $M_{2}^{0}\left(\theta, \Sigma^{1,0}, \Sigma^{1,0}\right) \cup \Sigma^{1,1,1,0} \cup M_{2}^{0}\left(\theta, \Sigma^{1,1,0}, \Sigma^{1,0}\right)$ and singular at points of $M_{2}^{0}\left(\theta, \Sigma^{1,0}, \Sigma^{1,1,0}\right) \cup M_{3}^{0}\left(\theta, \Sigma^{1,0}, \Sigma^{1,0}, \Sigma^{1,0}\right)$.

Let $z=\left([x], \ell_{0},[f]\right) \in M_{2}^{0}\left(\theta, \Sigma^{1,0}, \Sigma^{1,0}\right) \cup \Sigma^{1,1,1,0} \cup M_{2}^{0}\left(\theta, \Sigma^{1,1,0}, \Sigma^{1,0}\right)$. Then

$d \psi_{z}$ is not bijective $\Longleftrightarrow z \in \Sigma^{1,1,1,0}(\theta)$ or $x$ is singular on $V(f)$ (i.e. $\psi(z) \in \phi(\Delta)$ ).

Proof. The expression of the closure of $M_{2}^{0}\left(\theta, \Sigma^{1,0}, \Sigma^{1,0}\right)$ and smoothness at the points stated are general facts for maps with the transversality properties proclaimed in Proposition 3.1. One can check them on the explicit Example 1.2, where $K_{2}$ is empty.

If $z \in \Sigma^{1,1,1,0}(\theta), \operatorname{Ker}\left(d \theta_{z}\right) \subset T \bar{M}_{2}(\theta)_{z}$. Since $\psi$ is the composition $\bar{M}_{2}(\theta) \stackrel{\theta}{\rightarrow} W \stackrel{T}{\rightarrow} \mathbb{P}_{n}^{0}$, $\operatorname{Ker}\left(d \psi_{z}\right) \supset \operatorname{Ker}(d \theta)_{z}$, and so $d \psi_{z}$ is not bijective.

Let $\left.z_{0}=\left(\left[x_{0}\right], \ell_{0},[f]\right) \in M_{2}^{0}\left(\theta, \Sigma^{1,1,0}, \Sigma^{1,0}\right), \theta\left(z_{0}\right)=\theta\left(z_{1}\right), z_{1}=\left(\left[x_{1}\right], \ell_{0},[f]\right) \in \Sigma^{1,0}\right)$, $\left.\left[x_{0}\right] \neq\left[x_{1}\right]\right)$. We will use the local coordinates on $\mathbb{P}^{2} \times \check{\mathbb{P}}^{2} \times \mathbb{P}_{n}^{0}$, notation and expressions of tangent spaces to the various strata introduced in the proof of Proposition 3.1. Let $\bar{z}=(\bar{x}, 0,0) \in \operatorname{Ker}\left(d \theta_{z_{0}}\right) \cap \operatorname{Ker}\left(d \psi_{z_{0}}\right) ;$ since $z_{0} \in \Sigma^{1,1,0}, \bar{z} \in T\left(\Sigma^{1,0}\right)_{z_{0}}$ and $d \theta_{z_{0}}(\bar{z})=0$, and since $T\left(\bar{M}_{2}\right)_{z_{0}}=d\left(\theta \mid \Sigma^{1}(\theta)\right)_{z_{0}}^{-1}\left(d \theta_{z_{1}}\left(T\left(\Sigma^{1,0}(\theta)\right)_{z_{1}}\right)\right)$, we have that $\bar{z} \in T\left(\bar{M}_{2}\right)_{z_{0}}$, but $\bar{z} \in \operatorname{Ker}\left(d \psi_{z_{0}}\right)$.

If $\left.z_{0}=\left(\left[x_{0}\right], \ell_{0},[f]\right) \in M_{2}^{0}\left(\theta, \Sigma^{1,0}, \Sigma^{1,0}\right), z_{1}=\left(\left[x_{1}\right], \ell_{0},[f]\right) \in \Sigma^{1,0}\right),\left[x_{0}\right] \neq\left[x_{1}\right]$, then $\theta\left(z_{0}\right)=\theta\left(z_{1}\right)$ and

$\operatorname{Ker}\left(d S_{z_{0}}\right) \cap T\left(\Sigma^{1}\right)_{z_{0}}=\left\{(\bar{x}, \bar{\ell}, 0) \mid \bar{x} \in \ell_{0}, \bar{\ell}\left(x_{0}\right)=0, d^{2} f_{x_{0}}\left(\bar{x}, i_{\ell_{0}}\right)+d f_{x_{0}}(\bar{\ell})=0\right\}$

and

$$
\begin{aligned}
\operatorname{Ker}\left(d S_{z_{0}}\right) \cap d\left(\theta \mid \Sigma^{1}(\theta)\right)_{z_{0}}^{-1}\left(d \theta_{z_{1}}\left(T\left(\Sigma^{1,0}(\theta)\right)_{z_{1}}\right)\right)=\{(\bar{x}, \bar{\ell}, 0) \mid \\
\left.\bar{x} \in \ell, \bar{\ell}\left(x_{0}\right)=\bar{\ell}\left(x_{1}\right)=0, d^{2} f_{x_{0}}\left(\bar{x}, i_{\ell_{0}}\right)+d f_{x_{0}}(\bar{\ell})=0\right\}
\end{aligned}
$$

but $\bar{\ell}\left(x_{0}\right)=\bar{\ell}\left(x_{1}\right)=0 \Rightarrow \bar{\ell}=0 \Rightarrow d^{2} f_{x_{0}}\left(\bar{x}, i_{\ell_{0}}\right)=0 \Rightarrow \bar{x}=0$, which proves that $d \psi_{z_{0}}$ is injective.

If $\left.z_{0}=\left(\left[x_{0}\right], \ell_{0},[f]\right) \in \Sigma^{1}, z_{1}=\left(\left[x_{1}\right], \ell_{0},[f]\right) \in \Sigma^{1},\left[x_{0}\right] \neq\left[x_{1}\right]\right)$, and $d f_{x_{0}}=0$, then $z_{0} \in \bar{M}_{2}$; assume that $z_{0} \in M_{2}^{0}\left(\theta, \Sigma^{1,0}, \Sigma^{1,0}\right) \cup M_{2}^{0}\left(\theta, \Sigma^{1,1,0}, \Sigma^{1,0}\right)$. Then

$$
\left\{(0, \bar{\ell}, 0) \mid \bar{\ell}\left(x_{0}\right)=0\right\} \subset T\left(\Sigma^{1}(\theta)\right)_{z_{0}} \cap \operatorname{Ker}\left(d \theta_{z_{0}}\right) \subset T\left(\bar{M}_{2}\right)_{z_{0}}
$$

and so $\left\{(0, \bar{\ell}, 0) \mid \bar{\ell}\left(x_{i}\right)=0, i=0,1\right\} \subset \operatorname{Ker}\left(d \psi_{z_{0}}\right)$.

We prove now Theorem I. The control map for real flexes is $\phi_{\mathbb{R}}: \Sigma^{1,1}\left(\theta_{\mathbb{R}}\right) \rightarrow \mathbb{P}_{n, \mathbb{R}}^{0}$, whereas the control map for bitangents of type $t^{\prime \prime}$ is the restriction $\psi_{t^{\prime \prime}}: \bar{M}_{2}\left(t^{\prime \prime}\right) \rightarrow \mathbb{P}_{n, \mathbb{R}}^{0}$ of $\psi_{\mathbb{C}}$, where $\bar{M}_{2}\left(t^{\prime \prime}\right)=\bar{M}_{2}\left(\theta_{\mathbb{C}}\right) \cap\left(\left(\mathbb{P}_{\mathbb{C}}^{2} \backslash \mathbb{P}_{\mathbb{R}}^{2}\right) \times \check{\mathbb{P}}_{\mathbb{R}}^{2} \times \mathbb{P}_{n, \mathbb{R}}^{0}\right)$.

For $[f] \in \Omega_{n, \mathbb{R}}$, we denote by $w^{\prime}(f)$ the number of real flexes on $V(f)$ and by $t^{\prime \prime}(f)$ the number of its bitangents of type $t^{\prime \prime}$. 
Theorem 3.4. Let $\left[f_{0}\right],\left[f_{1}\right] \in \Omega_{n, \mathbb{R}}$. Then

$$
w^{\prime}(f)+t^{\prime \prime}(f)=w^{\prime}(g)+t^{\prime \prime}(g) .
$$

Proof. We can find a path in $\Omega_{n, \mathbb{R}}$ that misses the ramification loci of codimension not smaller than 2 of the control maps $\phi_{\mathbb{R}}$ and $\psi_{t^{\prime \prime}}$, and is transversal to those of codimension 1, namely:

1) Both for real flexes and bitangents of type $t^{\prime \prime}$ :

$$
\phi_{\mathbb{R}}\left(\Sigma^{1,1,1,0}\left(\theta_{\mathbb{R}}\right)\right)=\psi_{t^{\prime \prime}}\left(\Sigma^{1,1,1,0}\left(\theta_{\mathbb{R}}\right)\right) \quad \text { and } \quad \phi_{\mathbb{R}}\left(\Delta_{\mathbb{R}}^{0}\right) .
$$

2) For bitangents of type $t^{\prime \prime}$ only:

$$
\psi_{t^{\prime \prime}}\left(M_{2}^{0}\left(\theta_{\mathbb{C}}, \Sigma^{1,1,0}, \Sigma^{1,0}\right) \cap \bar{M}_{2}\left(t^{\prime \prime}\right)\right)=\psi_{t^{\prime \prime}}\left(M_{2}^{0}\left(\theta_{\mathbb{C}}, \Sigma^{1,0}, \Sigma^{1,1,0}\right) \cap \bar{M}_{2}\left(t^{\prime \prime}\right)\right)
$$

and

$$
\psi_{t^{\prime \prime}}\left(M_{3}^{0}\left(\theta_{\mathbb{C}}, \Sigma^{1,0}, \Sigma^{1,0}, \Sigma^{1,0}\right) \cap \bar{M}_{2}\left(t^{\prime \prime}\right)\right) .
$$

It follows from point 2) of Proposition 3.1 that $\phi_{\mathbb{R}}$ is a local homeomorphism near points of $\Delta_{\mathbb{R}}^{0}$, so $w^{\prime}$ does not change when $\phi_{\mathbb{R}}\left(\Delta^{0}\right)$ is crossed.

If $z=([x], \ell,[f]) \in M_{2}^{0}\left(\Sigma^{1,0}, \Sigma^{1,1,0}\right)$ and $\psi(z) \in \Delta^{0}$, then $[x]$ is a real ordinary double point of $f$ and $\ell$ is not tangent to the branches of $f$ through $[x]$, but it is simply tangent to a further real smooth point $\left[x_{1}\right] \in V(f)$. Nearby bitangents will be tangent at points $\left[x^{\prime}\right]$ and $\left[x_{1}^{\prime}\right]$, near to $[x]$ and $\left[x_{1}\right]$ respectively. Since $[x]$ and $\left[x_{1}\right]$ are not conjugate, neither are $\left[x^{\prime}\right]$ and $\left[x_{1}^{\prime}\right]$, and so $t^{\prime \prime}$ does not change in the neighborhood of $[f]$.

If $\left(\left[x_{1}\right], \ell,[f]\right) \in M_{2}^{0}\left(\theta_{\mathbb{R}}, \Sigma^{1,1,0}, \Sigma^{1,0}\right)$, let $\left[x_{2}\right] \neq\left[x_{1}\right]$ be the second point on $\ell$ tangent to $V(f)$. Since $\left[x_{1}\right]$ is a flex and $\left[x_{2}\right]$ is not, they cannot be complex conjugate, and the same holds for nearby bitangents. Therefore $\psi_{t^{\prime \prime}}\left(M_{2}^{0}\left(\theta_{\mathbb{C}}, \Sigma^{1,1,0}, \Sigma^{1,0}\right) \cap \bar{M}_{2}\left(t^{\prime \prime}\right)\right)$ is empty.

If $\left(\left[x_{1}\right], \ell,[f]\right) \in M_{3}^{0}\left(\theta_{\mathbb{C}}, \Sigma^{1,0}, \Sigma^{1,0}, \Sigma^{1,0}\right)$, let further $\ell$ be simply tangent to $V(f)$ at $\left[x_{2}\right],\left[x_{3}\right]$, with $\left[x_{1}\right],\left[x_{2}\right]$ and $\left[x_{3}\right]$ all distinct. If $\left[x_{1}\right]$ and $\left[x_{2}\right]$ are complex conjugate, then bitangents near $\ell$ at points near $\left[x_{1}\right]$ and $\left[x_{2}\right]$ will be of the same type, therefore $t^{\prime \prime}$ does not change when crossing $\psi_{t^{\prime \prime}}\left(M_{3}^{0}\left(\theta_{\mathbb{C}}, \Sigma^{1,0}, \Sigma^{1,0}, \Sigma^{1,0}\right) \cap \bar{M}_{2}\left(t^{\prime \prime}\right)\right)$.

It remains to examine what happens when we $\operatorname{cross} \phi_{\mathbb{R}}\left(\Sigma^{1,1,1,0}\left(\theta_{\mathbb{R}}\right)\right)$. For this we use the explicit Example 1.2:

$$
F\left(x_{1}, x_{2}, x_{3}\right)=\left(x_{1}, x_{2}, x_{3}^{4}+x_{3}^{2} x_{1}+x_{3} x_{2}\right) .
$$

As explained in Section 1, if $z \in \Sigma^{1,1,1,0}(\theta)$, in a suitable system of coordinates around $z$ and $\theta(z)$, the map $\theta$ reads:

$$
\left(x_{1}, x_{2}, x_{3}, u\right) \mapsto\left(F\left(x_{1}, x_{2}, x_{3}\right), u\right) .
$$

Recall the parametrizations:

$$
F\left(M_{2}\left(F, \Sigma^{1}, \Sigma^{1}\right)\right): \quad x_{3} \mapsto\left(-2 x_{3}^{2}, 0, x_{3}^{4}\right)
$$

and

$$
F\left(\Sigma^{1,1}(F)\right): \quad x_{3} \mapsto\left(-6 x_{3}^{2}, 8 x_{3}^{3}, x_{3}\right) .
$$

If we call $\left(X_{1}, X_{2}, X_{3}\right)$ the three coordinates in the target of $F$, we see that 
1) for $c<0$, the plane $X_{1}=c$ cuts $F\left(\Sigma^{1,1,0}(F)\right)$ transversally in the two distinct real points whose preimages by $F$ are

$$
\left(c, 8(\sqrt{-c / 6})^{3}, \sqrt{-c / 6}\right) \text { and }\left(c,-8(\sqrt{-c / 6})^{3},-\sqrt{-c / 6}\right)
$$

and it cuts $F\left(M_{2}\left(\Sigma^{1,0}, \Sigma^{1,0}\right)\right)$ transversally in one point, with two real preimages by $F$ : $(c, 0, \pm \sqrt{-c / 2})$

2) for $c>0$, the intersection of the plane $X_{1}=c$ with $F\left(\Sigma^{1,1,0}(F)\right)$ has no real points, and it cuts $F\left(M_{2}\left(\Sigma^{1,0}, \Sigma^{1,0}\right)\right)$ transversally in one point, with the two imaginary conjugate preimages by $F:(c, 0, \pm \sqrt{-c / 2})$.

It suffices now to show that the projection of the $X_{1}$-axis (of the target) on $\mathbb{P}_{n}^{0}$ is transversal to the stratum $\phi_{\mathbb{R}}\left(\Sigma^{1,1,1,0}\left(\theta_{\mathbb{R}}\right)\right)$. We have seen in the proof of Proposition 3.1 that for $z=\left(\left[x_{0}\right], \ell_{0},[f]\right) \in \Sigma^{1,1}(\theta) \backslash \Delta$ the tangent space $T \Sigma^{1}(\theta)_{z}$ can be expressed as

$$
\left\{(\bar{x}, \bar{\ell}, \bar{f}) \mid \bar{x}^{\prime}-\bar{\ell}\left(x_{0}^{0}\right)=0, \bar{f}\left(x_{0}\right)+d f_{x_{0}}(\bar{x}), d \bar{f}_{x_{0}}\left(i_{\ell_{0}}\right)+d^{2} f_{x_{0}}\left(\bar{x}, i_{\ell_{0}}\right)+d f_{x_{0}}(\bar{\ell})=0\right\} .
$$

If $(\bar{x}, \bar{\ell}, 0) \in T \Sigma^{1}(\theta)_{z} \cap \operatorname{Ker}\left(d S_{z}\right)$, where $S: W \rightarrow \mathbb{P}_{n}^{0}$ is the natural projection, then

$$
d f_{x_{0}}(\bar{x})=0 \Rightarrow \bar{x} \in \ell_{0} \Rightarrow \bar{x}^{\prime}=0 \Rightarrow \bar{\ell}\left(x_{0}\right)=0
$$

and

$$
\left.z \in \Sigma^{1,1} \Rightarrow d^{2} f_{x_{0}}\left(\bar{x}, i_{\ell_{0}}\right)=0 \Rightarrow d f_{x_{0}} \bar{\ell}\right)=0 \Rightarrow \bar{\ell}=0 .
$$

Therefore $T \Sigma^{1}(\theta)_{z} \cap \operatorname{Ker}\left(d S_{z}\right)=\ell_{0}=\operatorname{Ker}\left(d \theta_{z}\right)$. For the map $F$, a non-zero vector on the $x_{1}$-axis is contained in $T\left(\Sigma_{0}^{1}\right)$, but not in $\operatorname{Ker}\left(d F_{0}\right)$, and it is not tangent to $\Sigma^{1,1}$; the image of the $x_{1}$-axis by $d F_{0}$ is the $X_{1}$-axis. Therefore the projection the $X_{1}$-axis on $\mathbb{P}_{n}^{0}$ is transversal to $\phi_{\mathbb{R}}\left(\Sigma^{1,1,1,0}(\theta)\right)$. In conclusion, when crossing this stratum in the direction of increasing $X_{1}, w^{\prime}$ decreases by 2 and $t^{\prime \prime}$ increases by 1 , and so $w^{\prime}+2 t^{\prime \prime}$ remains unchanged.

It follows from the proofs of Lemma 3.2, Proposition 3.3 and of Theorem I that the structure of the control maps $\phi_{\mathbb{R}}$ and $\psi_{t^{\prime \prime}}$ near generic points of strata of codimension 1 does not depend on $n$, for $n \geq 3$. Therefore, in order to see how $w^{\prime}$ and $t^{\prime \prime}$ change when crossing a stratum of codimension 1, it is enough to work out explicit examples (a procedure used, or referred to, by Klein, sometimes implicitly). For $\phi_{\mathbb{R}}\left(\Delta_{\mathbb{R}}^{0}\right)$, we can use the family of cubics $f_{\alpha}(x, y)=y^{2}-(x+\alpha)^{2}(x-1), \alpha$ near 0 . For $\phi_{\mathbb{R}}\left(\Sigma^{1,1,1,0}\left(\theta_{\mathbb{R}}\right)\right)$, we have to use quartics, because $\Sigma^{1,1,1,0}\left(\theta_{\mathbb{R}}\right)=\emptyset$ for cubics; we can take $f_{\alpha}(x, y)=y^{2}-x^{4}+\alpha x^{2}$, $\alpha$ near 0 .

\section{Constructing examples of curves}

Remark 4.1. Let $[f] \in \mathbb{P}_{n, \mathbb{R}}^{0}$ be a real curve, $x_{1}^{0}$ and $x_{2}^{0}$ two real distinct ordinary double points on $V(f)$. Assume that the line $\ell$ through $x_{1}^{0}$ and $x_{2}^{0}$ is not tangent to the branches of $f$ through $x_{1}^{0}$ and $x_{2}^{0}$ and that it is not tangent to $f$ elsewhere. Then there exist arbitrary small neighborhoods $V$ of $[f]$ in $\mathbb{P}_{n, \mathbb{R}}^{0}$, and $U_{1}, U_{2}$ of $x_{1}^{0}, x_{2}^{0}$ respectively in $\mathbb{P}_{\mathbb{C}}^{2}$, with $U_{1} \cap U_{2}=\emptyset$ with the following property: if there exist $\left[f^{\prime}\right] \in V, x_{1} \in U_{1}, x_{2} \in U_{2}$ such that the line $\ell^{\prime}$ through $x_{1}$ and $x_{2}$ is tangent to $f^{\prime}$ at $x_{1}$ and $x_{2}$, then $\ell^{\prime}$ is not of type $t^{\prime \prime}$. Indeed, it suffices to choose $U_{1}$ and $U_{2}$ such that $x_{1}$ and $x_{2}$ cannot be conjugate. 
In fact, it is possible to show, more precisely, that under the above conditions, the control map $\psi: \bar{M}_{2} \rightarrow \mathbb{P}_{n}^{0}$ can be written in suitable local coordinates near $z=\left(x_{1}^{0}, \ell,[f]\right)$ as

$$
\left(x_{1}, x_{2}, \ldots, x_{N}\right) \mapsto\left(x_{1}^{2}, x_{2}^{2}, x_{3}, \ldots, x_{N}\right)
$$

but we do not need this here.

Now we will follow closely Klein's paper to construct examples of curves with the maximal number of real flexes.

Let first $n$ be even, $n=2 \mu$. In the affine real plane, take the ellipse $x^{2}+2 y^{2}=1$ and its images by rotations of an angle of $\pi / \mu \cdot h, h=0, \ldots, \mu-1$. The union of these ellipses is a curve $C_{n}$ of degree $n$, with $4 \mu\left(\begin{array}{c}\mu \\ 2\end{array}\right)=n(n-2) / 2$ real ordinary double points, and the lines joining two of them are not tangent to the other ellipses. When we deform the equation of $C_{n}$ slightly into $C_{n}^{\prime}$, double tangents can arise in three ways:

1) near lines joining pairs of distinct double points. They cannot be of type $t^{\prime \prime}$ by Remark 4.1.

2) near a line $\ell$ passing through a double point, intersection $x$ of two ellipses, and tangent to a third ellipse. Then $\left(x, \ell, C_{n}\right)$ is in $M_{2}^{0}\left(\theta, \Sigma^{1,0}, \Sigma^{1,0}\right)$ and we have seen in the proof of Theorem 3.4, by an argument similar to Remark 4.1, that there are no bitangents of type $t^{\prime \prime}$ nearby.

3) near a line $\ell$ tangent to distinct pairs of ellipses, at real points $x_{1}, x_{2}$. Then the derivative of $\psi$ at $z=\left(x_{1}, \ell, C_{n}\right)$ is bijective, and so nearby bitangents of nearby curves cannot be of type $t^{\prime \prime}$.

As a consequence, $t^{\prime \prime}\left(C_{n}^{\prime}\right)=0$.

According to point 2) of Proposition 3.1, applied to $\phi_{\mathbb{R}}$, each ordinary double point $x$ on $C_{n}$ gives rise to two real flexes near $x$ on $C_{n}^{\prime}$, one for each real branch through $x$. Therefore

$$
w^{\prime}\left(C_{n}^{\prime}\right)=2 \cdot 4 \cdot\left(\begin{array}{l}
\mu \\
2
\end{array}\right)=n(n-2) .
$$

This proves Theorem I for $n$ even. If $n=2 \mu+1$, we start with the same $\mu$ ellipses, but add a non-singular cubic which cuts each ellipse in 6 distinct points, not on the intersection of two ellipses. Such a cubic can be obtained as a small deformation of the cubic $y^{3}=0$. The union of the $\mu$ ellipses and the cubic form a curve $C_{n}$ of degree $n$. As before, a small deformation $C_{n}^{\prime}$ of $C_{n}$ will have $t^{\prime \prime}=0$, and $w^{\prime}\left(C_{n}^{\prime}\right)$ will be equal to twice the number of double points of $C_{n}$, plus the 3 real flexes of the cubic. Hence

$$
w^{\prime}\left(C_{n}^{\prime}\right)=2\left(4 \cdot\left(\begin{array}{l}
\mu \\
2
\end{array}\right)+6 \mu\right)+3=n(n-2) .
$$

This completes the proof of Theorem I.

For Theorem II, we first construct curves with the minimal number of flexes. $f_{2 \mu}=$ $\left(x^{2}+y^{2}+1\right)^{\mu}$ has no real points, and if $f_{3}$ is the equation of a real cubic, $f_{2 \mu+1}=f_{3} \cdot f_{2 \mu}$ has the same real points as the cubic. Small deformations of $f_{2 \mu}$ and $f_{2 \mu+1}$ provide smooth curves $g_{n}^{\min }$ without real flexes for $n$ even and with 3 real flexes for $n$ odd.

Denote by $g_{n}^{\max }$ the curves with maximal number of real flexes constructed in the proof of Theorem I and let $g_{t}^{n}, 0 \leq t \leq 1$ be a path in $\mathbb{P}_{n, \mathbb{R}}^{0}$, joining $g_{n}^{\min }$ to $g_{n}^{\max }$, which 
avoids the ramification loci of codimension $\geq 2$ of $\phi_{\mathbb{R}}$, the control map for real flexes, and transversal to those of codimension 1. Then, as in the proof of Theorem 3.4, when $g$ crosses a stratum of codimension $1, w^{\prime}$ can change by $2,-2$ or 0 . Therefore, as $t$ varies from 0 to $1, w^{\prime}$ must take all the values of the form $w^{\prime}=n(n-2)-2 k$, with $w^{\prime} \geq 0$ for $n$ even and $w^{\prime} \geq 3$ for $n$ odd.

\section{References}

[1] S. Akbulut (ed.), Real Algebraic Geometry and Topology, Contemp. Math. 182, Amer. Math. Soc., Providence, 1996.

[2] R. Benedetti and R. Silhol, Spin and Pin ${ }^{-}$structures, immersed and embedded surfaces and a result of B. Segre on real cubic surfaces, Topology 34 (1995), 651-678.

[3] M. Golubitsky and V. Guillemin, Stable Mappings and their Singularities, Springer, New York-Heidelberg 1973.

[4] F. Klein, Eine neue Relation zwischen den Singularitäten einer algebraischen Curve, Math. Ann. 10 (1876), 199-209.

[5] I. R. Porteous, Simple Singularities of Maps, preprint, Columbia Univ., 1962, reprinted in: Proc. of the Liverpool Singularities Symp., Lecture Notes in Math. 192, Springer, Berlin, 1971, 217-236.

[6] F. Sottile, Enumerative Geometry for real Varieties, to appear.

[7] R. Thom, Stabilité structurelle et morphogénèse, W. A. Benjamin, Reading, 1972.

[8] O. Ya. Viro, Some integral calculus based on Euler characteristic, in: Topology and Geometry - Rohlin Seminar, Lecture Notes in Math. 1346, Springer, Berlin, 1988.

[9] C. T. C. Wall, Duality of real projective plane curves: Klein's equation, Topology 35 (1996), 355-362.

[10] H. G. Zeuthen, Sur les différentes formes des courbes planes du quatrième ordre, Math. Ann. 7 (1874), 410-432. 